\title{
A PERSONALIZED SAFETY TRAINING SYSTEM FOR CONSTRUCTION WORKERS
}

\author{
S. Xu ${ }^{1,2^{*}}$ Q.Q. $\mathrm{Ni}^{3}$, Mengge Zhang $^{3}$ and Meiyan $\mathrm{Li}^{1}$ \\ ${ }^{1}$ School of Economics and Management, Chang'an University, Xi'an, China \\ ${ }^{2}$ International Joint Research Centre for Digital Construction and Management of Transportation Infrastructure along "Belt \\ and Road", Xi'an, China \\ ${ }^{3}$ School of Civil Engineering, Chang'an University, Xi'an, China \\ * Corresponding author
}

\begin{abstract}
Safety training for construction workers is believed to be an important approach to improve construction safety through reducing unsafe behaviour. Various research efforts have improved safety training by adapting training materials to workers' language capacities, applying information and communication technology in safety training to improve its efficiency, encouraging safety communication from foremen and co-workers, among others. However, limited research has been identified to analyse construction worker cognitive capabilities and improve their ability to learn. Therefore, this research proposed a personalized learning system to adapt safety training to every workers' learning ability and avoid the "one-size-fits-all" approach in safety training. Based on identified characteristics of construction workers' learning process, this research developed a recommendation algorithm to push training materials to workers according to their trades, workings activities, risk sources on site, and learning ability.
\end{abstract}

\section{Introduction}

The safety problem is highly concerned by the construction industry in many countries (Zahoor et al. 2016). In recent years, despite the progress in construction technology, construction management and construction environment (Choi et al. 2011), according to statistics from the United States, the United Kingdom and Hong Kong, the total number of accidents remains high and the number of fatalities in construction industry has not reduced significantly (Zhang and Fang 2016). This indicates that safety in the construction industry remains to be improved. Safety accidents can be the results of many factors (Garrett et al. 2009), of which human factors account for $70 \%$ (Haslam et al. 2005). Workers' unsafe behaviour has been proved by many accident investigations to be the major cause of accidents (Fang et al. 2016). As Wiegmann and Shappell (2001) pointed out, there is a causal relationship between human errors and accidents, and controlling human errors can reduce accidents. Therefore, it becomes urgent to solve how to reduce workers' unsafe behaviours.

On-site workers engaged in construction work are often under-educated, with inadequate safety knowledge and safety awareness of the safety risks caused by irregular operations. Thus, safety training for construction workers, which is used to effectively reduce unsafe behaviour, draws increasing attention by both researchers and project managers. As the scale of projects continues to expand and the working conditions become more complex, the requirements for the safety of workers at the construction site are also increasing (Pereira et al. 2018). Safety training usually takes place in a classroom or conference room onsite. The purpose is to prepare construction workers with adequate and proper safety awareness, safety knowledge, and safety skills, to meet the requirements of on-site safety management, and effectively prevent construction accidents. Studies have shown that trained workers are more safety-conscious and work more disciplined than those without proper training (Caban Martinez et al. 2018).

The positive role of safety training in enhancing knowledge and regulating behaviour has been recognized (Taylor, 2015), but accidents at construction sites frequently indicate that the training effect is not satisfactory, mainly due to the single training method and the boring training content. However, it is undeniable that the on-site safety management personnel on the construction site are inadequately equipped, work tasks are tight, training venues, time constraints, etc. These long-standing problems will directly lead to the difficulty of safety training work to be carried out and implemented as planned (Guo et al. 2016). Traditional safety training was mainly based on curriculum teaching and supplemented by text materials. It was not enough to attract workers, and a large amount of text is difficult for workers with low educational level to understand and remember in a short period of time. The series of real-world issues for safety training has forced project management to have an urgent need for a new type of safety training system.

Firstly, the content of training needs to be novel. The content of safety training mainly comes from operating procedures and safety regulations. The textual training materials are difficult to arise the interest of workers. Xu et al. (2014) 
transformed the normative regulations into vivid and interesting images, comics and photo-type training questions to be intuitive and easy to understand. Secondly, the training methods need to be diversified. A survey of 1197 lumberjack showed that video-based training can not only significantly enhance the safety knowledge of loggers, but also have a positive impact on safety attitudes and work habits (Helmkamp et al. 2004). Another study showed that the highparticipation training method can raise the risk awareness level of workers (Namian et al. 2016; Evanoff et al. 2016). Furthermore, training materials need to be comprehensive. In recent years, migrant workers have a high proportion in the US construction industry (Rathod, 2009) while taking up more casualties (Mayra De Jesus-Rivas et al. 2016). It is difficult for migrant workers to understand the Englishlanguage training materials. In order to overcome language barriers and ensure the effectiveness of training materials, Lin et al. (2018) developed a scenario-based training approach with 3D training materials for workers with low literacy and low English proficiency.

The variety of training schemes does not only transfer essential knowledge to workers, it also guarantees the attainment of certain task proficiency that is required to prepare workers for real-work task settings (Hou et al. 2017), as well as to instil a positive danger/risk-sensitive attitude and safety climate in the workplace (Shin et al. 2014; Choudhry 2014). Despite the ever-growing research focus on training techniques and methods, it is nonetheless important to investigate the effectiveness of and issues related to safety training from the training receivers' perspective, provided the recipients of safety training constitute heterogeneous workers. To this end, a personalised training schema can adapt to workers' learning characteristics and personal needs, and the most important component of constructing a personalised training schema should be characterising the heterogeneity of construction workers.

\section{Research Framework}

\subsection{Proposed framework of a personalized safety training system}

The personalised safety training system was consisted of three components, namely the training resource library, construction workers' learner model, and personalized resource recommendation algorithm, as shown in Figure 1.

\subsection{Semantic-based safety knowledge discovery}

Safety knowledge was not only derived from various safety standards, safety regulations, etc., but also in the unsafe behaviour of on-site construction operations. Therefore, safety training materials were diverse presentation of safety knowledge. Cognitive psychology suggested that coded verbal knowledge was learnt by storing the verbal information in schemas, and to assist the built of safety knowledge schemas could improve the efficiency of safety knowledge learning. A schema is a set of interrelated concepts of objects, events, or activities that revealed the structure of knowledge.
Figure 1 Framework of the proposed personalised safety training system

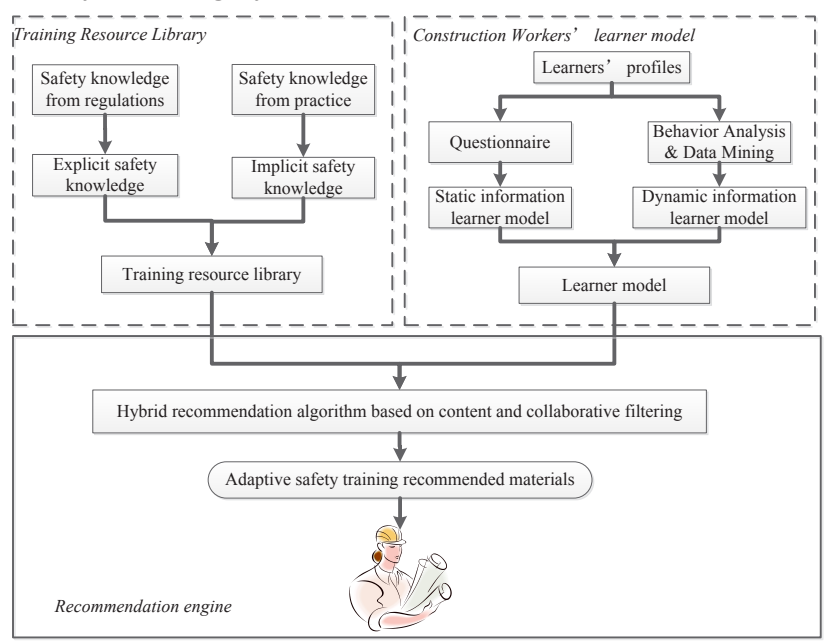

The structure of cognitive schemas was built around the core concepts. Construction activities were the process of interaction between workers and objects. The objects here included construction tools, materials, engineering objects, and environmental objects. In this way, workers and objects were set as the core concepts, and safety knowledge could be organised around them, describing the relationship between the core concepts in the construction context, it could improve the safety risks of construction workers in the construction process and environment, and avoid unsafe behaviour.

The construction safety schemas were constructed from safety regulations and specifications, and further integrated with knowledge from practice, or patrol data. It was first necessary to analyse and extract the behavioural safety knowledge in specifications, identify the core concept, and extract the safety attributes expressed in the specification around the core concept, as well as the mutual relations.

Secondly, in the construction practice, workers' unsafe behaviours were mostly reflected and captured by patrol photos or cameras. The patrol photos were the visualised representation of implicit safety knowledge. In order to integrate it into textual safety knowledge, safety managers were required to assign semantic information in the collection of photos, including core concepts, operational behaviours, operational objects, location areas, etc.

Finally, the on-site unsafe behaviours in 11 types were summarized, including safety protection (01), lifting and hoisting (02), earthwork (03), scaffolding (04), formwork engineering (05), high-altitude operation (06), fire safety (07), construction equipment (08), construction electricity (9), vehicle transportation (10), and other (11), as shown in Figure 2. Among them, taking various types of machinery into account, the lifting and hoisting (02) was subdivided into tower crane (021), gantry crane (022), material hoist (023). Similarly, construction equipment (08) is also subdivided into electric welder (081), smashing machine (082), steel 
machinery (083), hand-held power tools (084), concrete vibrator (085), and saws (086); and vehicles were divided into concrete mixers (101), excavator (102) and dumper (103), according to the professionalism.

Figure 2 Classification of construction workers' unsafe behaviour

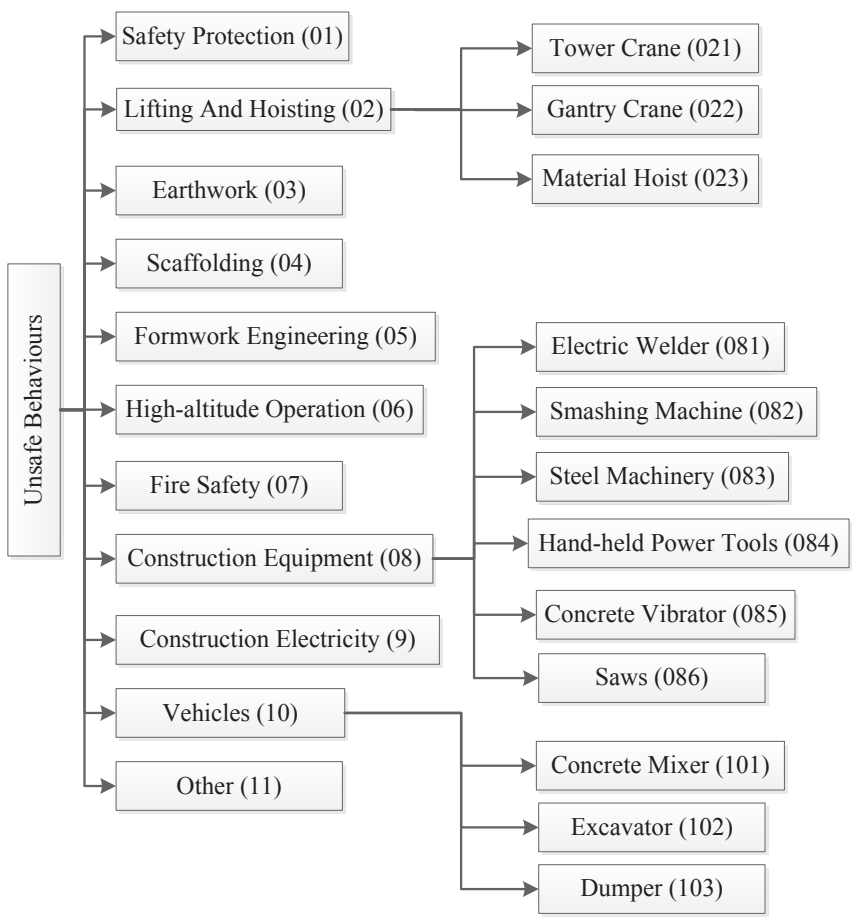

\subsection{Construction workers' learner model}

Describe factors of construction workers' learning process and their evaluation, the possible outcome of the evaluation of construction workers' learning process

Customisation/personalisation models in e-learning are based on the Learner Model which describes student characteristics and abilities for learning materials to be adapted to (Labib et al. 2017). Construction workers tended to have lower educational attainment and were more mobile than other industries. Therefore, a better knowledge of construction workers was more conducive to improving the effectiveness of safety training. This research herein developed the Learner Model for construction workers to obtain a taxonomy to achieve personalised safety training. The data of the Learner Model was based on evaluations gathered from a questionnaire-based survey.

At the time of learning, internal and external factors could collectively affect the attainment of knowledge. Learners' motivation could directly affect their determination and willingness to learn. According to the previous research, learners' willingness to learn relates to safety awareness, job requirements and workers' value judgments on safety training (Kuan-Chung Chen \& Syh-Jong Jang, 2010). The increase in safety awareness and workers value judgment might result in the improvement of their motivation, whereas job requirements, if mandatory, may demotivate them.
Emotions are people's reactions to objective things. The control of emotions can directly affect workers' behaviour and work efficiency. Darban and Polites (2016) highlighted that emotions could affect one's willingness to learn.

Previous experience referred to a worker's cognitive and kinetic experience developed from the past. It was the most dynamic factor that determines the learner's mastery of safety knowledge. Due to the variation of mobility and education, the background knowledge of each construction worker could be different. Furthermore, an unsafe behaviour that had not been promptly corrected could affect workers' safety perception, which could then influence the acceptance of knowledge during safety training.

Researchers purported that organisational culture can affect not only the worker's decision to share and communicate engineering-related knowledge (Ajmal, M.M., Koskinen, K.U., 2008) but also limit the training knowledge and creative imitation (Eskerod, P., Skriver, H.J., 2007). Han (2015) suggested that construction workers' working skills were mainly developed by learning and imitating from their workmates, which means a 'model' workmate could have a great impact on shaping his/her followers' behaviours.

During the construction process, construction workers are likely to act unsafely because absolute compliance to safety measures may sometimes compromise efficiency and convenience. Therefore, the intention of pursuing work efficiency with compromised safety standards could be a common factor in hazardous incidents.

\section{The Recommendation Method}

\subsection{Commonly used recommendation algorithms}

The adaptive safety training system was to recommend appropriate training resources by matching them with construction workers' safety learning needs. After 20 years of development, the recommendation algorithms used different fields of knowledge to improve the recommendation efficiency from multiple perspectives. At present, the most used algorithms included content-based filtering, association rule-based recommendation, collaborative filtering, and mixed recommendation.

The content-based filtering was the most basic information filtering algorithm, firstly proposed by Lang et al. in 1995. It was to filter the irrelevant information and extract the information of interest to users by matching the resource with the user interest model. The advantages of the content-based filtering algorithm were that it barely depends on users' evaluation of resources, and the algorithm was highly targeted. The disadvantage was that it was only applicable to the recommendation of text resources. It first identified users' personal information with their interest model, or the user vector; then filtered the text resources with machine learning, probability statistics or other techniques. Finally, it calculated the similarity of the established user and resource vector models, and the top $\mathrm{N}$ with high similarity was recommended 
to the user. The CF-IDF method proposed by Goossen et al. used cosine similarity to measure the similarity between users and projects.

The association rule-based recommendation is to measure the relevance between the items based on the analysis of user's current interests according to rules customized by the administrator. The recommendation system based on association rules has two shortcomings. Firstly, the accuracy of the recommendation is not guaranteed, because rules are manually customized by the administrator and not automatically generated by the system. Secondly, once the rules are formulated, they cannot change dynamically.

Collaborative filtering recommendation is one of the most widely used recommendation algorithms. It is to find similar users to recommend goods or information to the target users by collecting user evaluations on the resources and inferring target users' interests with the evaluation of similar users. Therefore, the most important step in collaborative filtering technology is to calculate the similarity among users and find the nearest neighbour. Collaborative filtering can provide better recommendations for users with relatively dense scoring data without a fully understanding of user's information. However, users' evaluation on resources has to be acquired in advance. When the scoring matrix is sparse, the recommendation effect is greatly reduced. The collaborative filtering algorithm is divided into three steps, the first step is to acquire and analyse user information, and the second step is to find the neighbour by calculating the similarity between the target users, thereby generating recommendations. The third step is to score predictions and generate recommendations.

In summary, the collaborative filtering and the content-based two recommendation algorithms are the classic algorithms of the recommendation system, but they have shortcomings individually. Organically mixing the two classic algorithms to form a new hybrid recommendation algorithm is possible to improve recommendation quality. Therefore, this research proposed a hybrid recommendation algorithm that combines content-based filtering and collaborative filtering.

\subsection{The proposed hybrid recommendation method}

To solve the "cold start" problem, the hybrid recommendation algorithm used content-based recommendation as a starter. When adequate data on users' preference and evaluations on learning resources were gathered, the personalised learning system applied the collaborative filtering method to generate recommendations. The process of proposed hybrid recommendation method was shown in Figure 3.
Figure 3 The proposed hybrid recommendation method

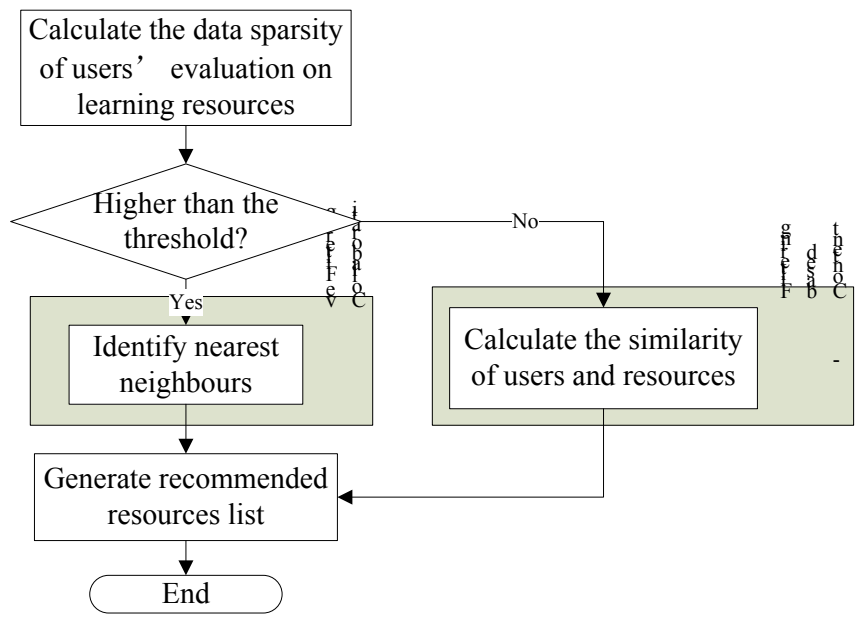

Step 1 Content-based filtering as a starter

In the content-based filtering, top $\mathrm{N}$ resources with the highest similarity were recommended to users. The following formula was used to calculate the similarity between the user model and the resource model for the match of users and resources,

$\sin (\vec{u}, \vec{r})=\cos (\vec{u}, \vec{r})=\frac{\vec{u} \cdot \vec{r}}{\|\vec{u}\|,\|\vec{r}\|}$

Note that the content filtering to generate resource recommendations for new registered users. Therefore, the user's recommendation in the system does not need to start from zero. After the user accessed the recommended resources generated by the content filtering, the resource could be scored.

\section{Step 2 Compare data sparsity with the threshold}

A threshold on data sparsity value was set. When the data sparsity was less than the threshold, the content filtering correction collaborative filtering algorithm was selected. When the score reached the threshold, collaborative filtering recommendation would be performed.

\section{Step 3 Identify the nearest neighbours}

After establishing the user-resource scoring matrix, the similarity between users could be calculated to form a neighbour set. By obtaining the similarity between users with Equation 1, the first $n$ users who took the greatest similarity formed neighbours, participated in predictive scoring, and generated recommendations.

\section{Step 4 Generate recommendations}

After obtaining the nearest neighbour of the target item by the method proposed above, the next step is to generate a corresponding recommendation. Let the nearest neighbour set of the target item TI be represented by $N N_{T I}=$ $\left\{N N_{1}, N N_{2}, \cdots, N N_{k}\right\}$, then the predictor score $\mathrm{P}_{u, T I}$ of the learner $u$ for the item TI could be obtained by the learner $\mathrm{u}$ 
scoring the items in the nearest neighbour set $N N_{T I}$, and the calculation method was shown in Equation 2.

$\mathrm{P}_{u, T I}=\overline{R_{T I}}+\frac{\sum_{n \in N N_{T I}} \operatorname{sim}(T I, n) *\left(R_{u, n}-\overline{R_{n}}\right)}{\sum_{n \in N N_{T I}}(|\operatorname{sim}(T I, n)|)}$

in which $\operatorname{sim}(T I, n)$ represented the similarity of the target $T I$ and nearest neighbour $n, R_{u, n}$ represented the score of $n$ given by user $u$, and $\overline{R_{T I}}$ and $\overline{R_{n}}$ represented the average score on $T I$ and $n$.

\section{Conclusion}

This paper proposed a personalised safety training system for construction workers. The system integrated safety knowledge in safety regulations and practice and organised them in safety knowledge schemas so that the training materials could be easily tied to construction contexts and understood. On the other hand, this paper argued that construction workers were heterogeneous and their learning characteristics could be described with learner model in dimensions of motivation, emotion, previous experience, model effect and convenience effect. Based on the classification of safety knowledge and construction workers, this research proposed a hybrid recommendation method to adaptively recommend training materials to construction workers, which combined content-based filtering and collaborative filtering algorithms together.

\section{Acknowledgements}

This work was supported financially by the National Natural Science Foundation of China (51708039), and Shaanxi Province Science Foundation (2018SD0018).

\section{References}

Ajmal, M.M.et al. (2008). Knowledge transfer in projectbased organizations: an organizational culture perspective. Proj. Manag. J. 39(1): 7-15.

Caban-Martinez, A. J. et al. (2018). Physical exposures, work tasks, and osha-10 training among temporary and payroll construction workers. Journal of Occupational and Environmental Medicine, 60(4): e159-e165.

Chan Albert P.C. et al. (2016) Strategies for Improving Safety and Health of Ethnic Minority Construction Workers. J. Constr. Eng. Manage., 142(9): 05016007.

Chen, K.C.et al. (2010). From PCK to TPACK: developing a transformative model for pre-service science teachers. Journal of Science Education \& Technology, 19(6): 553-564.

Choi, T. N. Y. et al. (2011) Perceived benefits of applying pay for safety scheme (PFSS) in construction - a factor analysis approach. Safety Science, 49(6): 813-823.

Choudhry, R.M., (2014) Behaviour-based safety on construction sites: A case study. Accident Analysis \& Prevention, 70: 14-23.
Eskerod, P.et al. (2007). Organizational culture restraining in-house knowledge transfer between project managers-a case study. Proj. Manag.J. 38(1): 110-122.

Evanoff, B. et al. (2016). Results of a fall prevention educational intervention for residential construction. Safety Science, 89: 301-307.

Fang, D. et al. (2016) A cognitive model of construction workers' unsafe behaviours. J. Manage. Eng., 142: 04016039.

Fang, D et al. (2016) A cognitive model of construction workers' unsafe behaviours. Journal of Construction Engineering and Management, 142, 04016039.

Garrett, J.et al. (2009) Student attitudes towards the use of graphical programming languages in an introductory engineering course. Computers in Education Journal, 19(01):60-69.

Guo, B.H.W. et al. (2016) Predicting safety behaviour in the construction industry: Development and test of an integrative model. Safety Science, 84, 1-11.

Han Y et al. (2015). Shaping strategies and methods of safety behaviour habits for construction workers. Journal of Safety Science and Technology, 09(11): 177-18.

Haslam, R.A. et al. (2005) Contributing factors in construction accidents. Applied Ergonomics, 36(4): 401-415.

Helmkamp, J.C. et al.(2004) Assessing safety awareness and knowledge and behavioural change among West Virginia loggers. Injury Prevention (1353-8047), 10(4): 233.

Hou, L.et al. (2017) A framework of innovative learning for skill development in complex operational tasks. Automation in Construction, 83: 29-40.

Jesus-Rivas, M.D. et al. (2016) The impact of language and culture diversity in occupational safety. Workplace Health \& Safety, 64(1): 24-27.

Labib, A.E.et al. (2017). On the way to learning style models integration: a learner's characteristics ontology. Computers in Human Behaviour, 73: 433-445.

Lin K et al. (2018) Training of Low-Literacy and LowEnglish-Proficiency Hispanic Workers on Construction Fall Fatality. J. Manage. Eng., 34(2): 05017009.

Mehdi Darban, et al.(2016). Do emotions matter in technology training? Exploring their effects on individual perceptions and willingness to learn. Computers in Human Behaviour, 62: 644-657.

Namian M et al. (2016) Improving Hazard-Recognition Performance and Safety Training Outcomes: Integrating Strategies for Training Transfer. J. Constr. Eng. Manage., 142(10): 04016048.

Portugal-Pereira, J. et al. (2018). Better late than never, but never late is better: risk assessment of nuclear power construction projects. Energy Policy, 120: 158-166.

Rathod, S.et al. (2009) Cognitive behaviour therapy across cultures. Psychiatry, 8(9), 370-371.

Shin, M. et al. (2014) A system dynamics approach for modeling construction workers' safety attitudes and behaviors. Accident Analysis \& Prevention, 68: 95-105. 
Suraji, A.et al. (2001) Development of causal model of construction accident causation. Journal of Construction Engineering and Management, 127(4): 337-344.

Taylor et al. (2015) Safety benefits of mandatory OSHA 10h training. Safety Science, 77: 66-71.

Wiegmann, D. A.et al. (2001). Human error analysis of commercial aviation accidents using the human factors analysis and classification system (HFACS). Aviation Space \& Environmental Medicine, 72(11): 1006.

$\mathrm{Xu} \mathrm{S}$ et al. (2014) A Behaviour Safety Training System Based on Visual Language. Journal of Civil Engineering and Management, (3): 51-55.

Zahoor, H. et al. (2016). Occupational safety and health performance in the Pakistani construction industry: stakeholders' perspective. International Journal of Construction Management: 1-11. 